\title{
GAME EFFICIENCY OF ELITE FEMALE WHEELCHAIR BASKETBALL PLAYERS DURING WORLD CHAMPIONSHIPS (GOLD CUP) 2006
}

\section{Bartosz Molik*, Andrzej Kosmol", Natalia Morgulec-Adamowicz", James J. Laskin ${ }^{* *}$, Tomasz Jezior*, Michał Patrzałek*}

\author{
* Jozef Pilsudski University of Physical Education in Warsaw, Faculty of Rehabilitation, Warsaw, \\ Poland \\ ** University of Montana, School of Physical Therapy and Rehabilitation Science, Missoula, \\ Montana, USA
}

\begin{abstract}
The aim of this study was two-fold: (1) to describe of game efficiency in relation to teams ranking in Gold Cup 2006, and (2) to explore the relationship between the functional classification levels of female elite wheelchair basketball athletes and their basketball performance. All 72 athletes representing the eight teams participating in the World Championships for Wheelchair Basketball in 2006 (3-16 July, Amsterdam, the Netherlands) were evaluated in this study. All twenty-four scheduled games were videotaped. Female athletes were divided according to their functional classification level $\left(1,1.5,2,2.5,3,3.5,4\right.$, and 4.5) and Gold Cup's team ranking (teams from $1^{\text {st }}$ to $4^{\text {th }}$ place, teams from $5^{\text {th }}$ and $6^{\text {th }}$ place, teams from $7^{\text {th }}$ and $8^{\text {th }}$ place). Nineteen variables of game efficiency were evaluated. To determine quality of an athlete's contribution to the game, modification of the Comprehensive Basketball Grading System (CBGS modified $_{\text {) was }}$ used. Analyses of the results demonstrated that game efficiency in women's wheelchair basketball is dependent on the athlete's functional level and team ranking. The results support the notion that athletes from the "best" teams demonstrate higher shooting efficiency and significantly better CBSG values in comparison to female players from the "weakest" teams. In addition the data shows many similarities in game efficiency between those athletes from adjacent classifications levels.
\end{abstract}

KEYWORDS: wheelchair basketball, game efficiency, functional classification.

\section{INTRODUCTION}

Among the competitive team sports that are available to athletes with physical disabilities, wheelchair basketball is the most developed in terms of number of participants, spectators' understanding, organization, standardization of rules and classification, as well as the quality of coaching. Wheelchair basketball is played on a recreational and competitive level in more than 80 countries worldwide. Over the past 60 years wheelchair basketball has evolved from a rehabilitative tool to a significant competitive sport.

In 1982 the functional classification system was adopted by the International Wheelchair Basketball Federation (IWBF) and has been used worldwide except in the United States. Wheelchair basketball athletes are divided into five main classes: 1, 2, 3, 4 and 4.5 ( 4.5 being characterized as minimal disability) and three subclasses 1.5, 2.5, and 3.5 used for athletes meeting the inclusion criteria of two adjacent classes (Courbarioux, 1996; IWBF, 2004). To ensure that all athletes, regardless of their functional capacity $^{1}$ have an equitable opportunity to participate in wheelchair basketball the sum of points (classification level) of the five players on the court cannot be greater than 14 points. Trained classification officers are responsible for the process of IWBF functional classification through a systematic

\footnotetext{
${ }^{1}$ In present study authors followed terminology that was used in A Guide to the Functional Classification of Wheelchair Basketball Players (IWBF 2004)
} 
method of off-court evaluation and observation of the athlete during play.

Various performance and physiological criteria have been used to evaluate the intended discrete nature of each of the eight functional classification levels. Numerous authors have examined aerobic and anaerobic performance (Hutzler, Ochana, Bolotin \& Kalina, 1998; Molik, Kosmol \& Rutkowska, 2005; Rotstein et al., 1994; Schmid et al., 1998; Vanlandewijck, Spaepen \& Lysens, 1995). Many have used standardized sportspecific skill tests (Brasile, 1990; Brasile \& Hedrick, 1996; Doyle et al., 2004). Malone, Gervais, and Steadward (2002) looked at shooting mechanics to differentiate players' performance. Game efficiency ${ }^{2}$ or game performance criteria such as field goals, rebounds, assists, and blocked shots have also been used to evaluate athletes' performance across classification levels (Molik \& Kosmol, 2001; Vanlandewijck et al., 1995; Vanlandewijck et. al., 2003; Vanlandewijck et al., 2004). Interestingly, most of these studies did not show significant differences between classes, except for those athletes with the lowest functional abilities. There were underlined similarities between class 2 and class 3 underlined in previous researches, also. Authors found significant differences between all other main classes (Molik \& Kosmol, 2001; Vanlandewijck et. al., 2003; Vanlandewijck et al., 2004). However, Vanlandewijck et al. (1995) used more research methods and proposed amalgamation classes from 2 to 4 . Thus, a majority of these authors have suggested that an amalgamation of classes and/or a re-examination of the current classification system is warranted. A clear dissenting voice to this suggestion has been through the work of Vanlandewijck et al. (2003, 2004). These authors have demonstrated a clear relationship between players; positioning and functional classification attributed to elite male and

\footnotetext{
${ }^{2}$ Efficiency is the positive estimation of an action and the goal of that action. The action is efficient if it enhances to realization of the goal (Pszczolowski 1978).
}

female athletes and their sport-specific performance. They evaluated game efficiency of male and female athletes during the World Championships (Gold Cup) 1998. Vanlandewijck et al. (2004) concluded that the IWBF functional classification system adequately differentiates between athletes of differing classification levels. However, in all the previous research only main classes were compared without the inclusion of the sub classes.

Two studies have specifically addressed the relationship between game efficiency and classification level in female wheelchair basketball athletes. Vanlandewijck et al. (2004) showed that the athletes' level of performance correlated with their classification level and position (guard, centre and forward). Schmid et al. (1998) analysed competitive games played by the German women' national team. These authors reported that athletes reached level of performance (players' performance diagram was established based on rebounds, points marked, and forced turnovers in defence) in accordance with their functional capacity. A weakness of both of these studies is that only the main classification levels were evaluated versus including the three subclasses. Both studies also evaluated game efficiency on games played prior to some significant IWBF rule changes. For instance in 2000, ten seconds rule was instead by eight seconds rule ("if the possession of the ball changes in the backcourt; the ball must cross into the frontcourt within the first 8 seconds of the possession"), and thirty seconds rule was instead by twenty-four seconds rule ("whenever a player gains control of a live ball on the playing court, his team must attempt a shot for a field goal within twentyfour seconds") (IWBF, 2008). The IWBF functional classification system has also undergone some "liberalization". For instance, athletes with hemipelvectomy have been reclassified from class 4 to 3.5 , and those with bilateral above knee amputees have been reclassified from 3 to 2.5. Moreover, players with new strapping 
shouldn't change their classification to higher classes like before.

The aim of this study was two-fold: (1) to describe of game efficiency in relation to teams ranking in Gold Cup 2006, and (2) to explore the relationship between the functional classification levels of female elite wheelchair basketball athletes and their basketball performance. We believe that there is a relationship between game efficiency and team ranking (Gold Cup 2006) in female' wheelchair basketball. We hypothesized that there is a relationship between game efficiency and functional capabilities of elite women wheelchair basketball athletes.

\section{METHOD}

\section{Participants}

A potential group of 94 elite female athletes representing eight teams participating in World Championships for Wheelchair Basketball (Gold Cup) in 2006 (3-16 July, Amsterdam, the Netherlands) took part in this study. All twenty-four scheduled games were videotaped. Each team was videotaped for six 40-min games for a total of $240 \mathrm{~min}$ per team. To be included in the analysis; an athlete had to play for a combined total of at least $40 \mathrm{~min}$ and participated in a minimum in 4 games during tournament. Seventy-two athletes met this criteria were divided according to their functional classification level $(1,1.5,2,2.5$, 3, 3.5, 4, and 4.5) and Gold Cup's team ranking.

\section{Table 1}

Number of female wheelchair basketball athletes per functional level (classification) and group (team ranking)

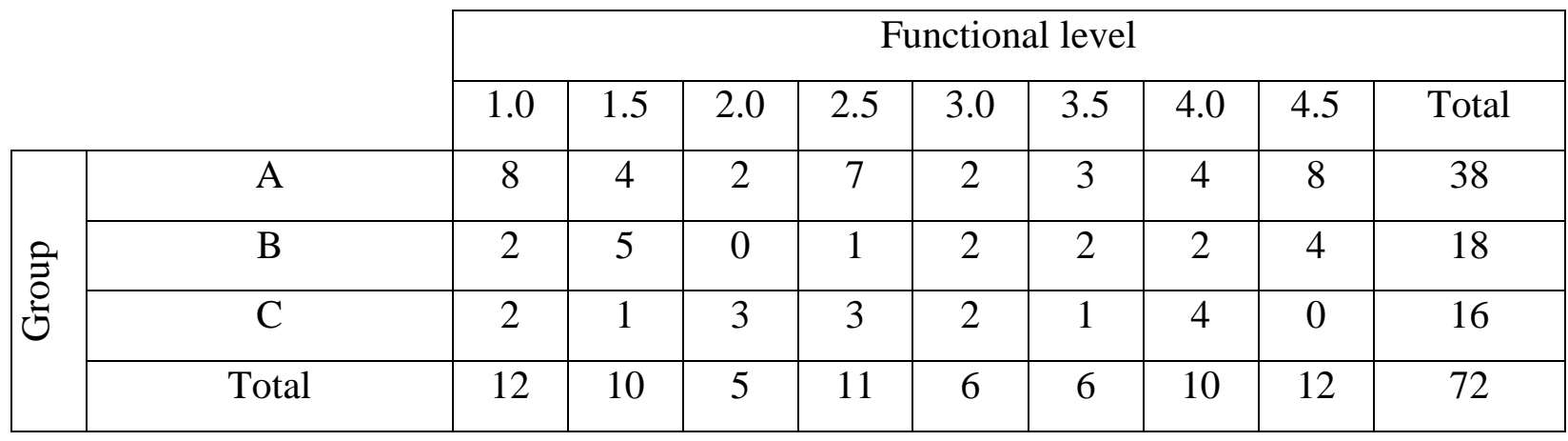

Note:

A-teams from $1^{\text {st }}$ to $4^{\text {th }}$ place, $B$ - teams from $5^{\text {th }}$ and $6^{\text {th }}$ place, $C$ - teams from $7^{\text {th }}$ and $8^{\text {th }}$ place.

Teams were grouped according to ranking into three groups: A (teams from $1^{\text {st }}$ to $4^{\text {th }}$ place), group B (teams from $5^{\text {th }}$ and $6^{\text {th }}$ place), and group $\mathrm{C}$ (teams from $7^{\text {th }}$ and $8^{\text {th }}$ place). All coaches and team managers were informed about the research study during team managers meeting prior to the competition. The study was approved by Jozef Pilsudski University of Physical Education's (Warsaw, Poland) Senate's Commission of Science Research Ethics.

\section{Protocol}

Two wheelchair basketball and running basketball experts independently scored all 24 games with a third expert (with 15 years of experience in wheelchair basketball) scoring a random selection of four games. A total of 19 variables related to critical game elements were evaluated: total points scored (TP), total shots attempt (TSa), total shots made (TSc), shots from 3 second area attempt (BSa), shots from 3 second area made (BSc), two-point shots attempt (S2a), two-point shots made (S2c), three-point shots attempt (S3a), threepoint shots made (S3c), free throws attempt (FTa), free throws made (FTc), steals (ST; when the defensive player gains ball possession due to an intentional activity, this can be caused either by a ball handler mistake 
or by aggressive defense. The player who gains possession of the ball is always responsible for it. A steal is connected with an attacking player's loss of possession. There was no steal recorded if the ball became dead without a turnover occurring), assists (AS; the last ball passed that creates a score. There cannot be more than one assist to one score. The most important fact is if the offensive player who received the pass scores), blocked shots (BL), offensive rebounds (OR), defensive rebounds (DR), all cause turnovers (TO; loss of ball possession. The activity of an offensive player that leads to a defender gaining possession of the ball. Although it can be also caused by the defender's activity), points scored after fast break (FA; points scored after a fast action, before defenders have the time to take their positions), and points scored directly after offensive rebounds by rebounder (DO). These 19 variables allow for the evaluation of the individual athletes' performance on the court.

To determine quality of an athlete's contribution to the game, the modification of Comprehensive Basketball Grading System ( $\left.\mathrm{CBGS}_{\text {modified }}\right)$ was used. The CBGS system was adopted for wheelchair basketball by Byrnes and Hedrick (1994), and implemented by Vanlandewijck et al. (2003, 2004). To evaluate the quality of game performance, the following factors were considered in the CBGS: offensive rebounds (+4), defensive rebounds $(+4)$, steals $(+5)$, blocked shots $(+5)$, assists $(+5)$, turnovers $(-6)$, personal fouls $(-$ $2)$, technical fouls $(-10)$, back picks $(+4)$, free throws made $(+4)$, free throws missed $(-2)$, two-point shots made $(+5)$, two-point shots missed (-3), three-point shots made $(+6)$, three-point shots missed (-4), forced turnovers when on defense (+6). Of these 16 , back picks, forced turnovers in defense, and both fouls were not used as there was a misunderstanding in interpretation those variables among our experts. Finally in CBGS $_{\text {modified }} 12$ from 16 variables were used.

There was high inter-observer reliability across observers for each variable, coefficients varying between $r=.85$ to $r=$ 1.00. All matches were also verified with official games score sheet and games statistics prepared by tournament organizers directly after each game.

\section{Data analysis}

Time of playing on the court for each player was documented. Each athlete's absolute score for the 19 measured variables was recalculated in a relative score format per 40 minutes. Number of actions per game was analyzed. Shooting efficiency rates between attempts and made shoots were calculated into percentage values. The following shooting efficiency rates were calculated: total shooting efficiency (TS\%), shots from 3 second area efficiency (BS\%), two-point shot efficiency (S2\%), three-point shot efficiency (S3\%), free throw efficiency (FT\%).

The $\mathrm{CBGS}_{\text {modified }}$ for each player was calculated using the following equation:

$\mathrm{CBGS}_{\text {modified }}=(\mathrm{OR} \times 4)+(\mathrm{DR} \times 4)+(\mathrm{ST} \times$ $5)+($ BL x 5) $+($ AS x 5) $+($ FTc x 4) $+($ S2c x $5)+($ S3c x 6) $-[($ FTm x 2) $+($ S2m x 3) + $(\mathrm{S} 3 \mathrm{~m} \times 4)+(\mathrm{TO} \times 6)]$

where $\mathrm{OR}=$ offensive rebounds, $\mathrm{DR}=$ defensive rebounds, $\mathrm{ST}=$ steals, $\mathrm{BL}=$ blocked shots, AS = assists, FTc $=$ free throws made, $\mathrm{S} 2 \mathrm{c}=$ two-point shots made, $\mathrm{S} 3 \mathrm{c}=$ three-point shots made, FTm $=$ free throws missed, $\mathrm{S} 2 \mathrm{~m}=$ two-point shots missed, $\mathrm{S} 3 \mathrm{~m}=$ three-point shots missed, and TO = turnovers.

Data were analyzed using SPSS (14.0). Descriptive statistics (mean and standard deviation) were calculated for each teamranking group (A, B and C) and for the eight functional classification levels (classes from 1.0 to 4.5 ). To examine differences between groups one-way analysis of variance (ANOVA) was performed. Tamhane's posthoc analysis was used to determine where among the group differences occurred. Spearman rank order correlation coefficients were computed to evaluate relationships between game efficiency variables, and $\mathrm{CBGS}_{\text {modified }}$ values (quality of contribution). Statistical significance was set at $\mathrm{p}<.05$. 


\section{RESULTS}

One-way analysis of variance (ANOVA) indicated significant differences among the three team ranking groups in total shots $F(2$, $69)=2.53, p=.087$; two-point shots, $F(2,69)$ $=4.02, p=.022$; free throws attempts, $F(2$, $69)=4.43, p=.015$; and quality of the game $\left(\mathrm{CBGS}_{\text {modified }}\right), F(2,69)=5.62, p=.005$. Tamhane's post-hoc analyses revealed significant differences between groups $\mathrm{A}$ and C for two-point shots $(37.51 \%$ and $24.81 \%$, respectively), total shots $(36.14 \%$ and 24.39 $\%$, respectively) and quality of the game (37.93 and 8.91, respectively). A significant difference was also observed between groups $\mathrm{A}$ and $\mathrm{B}$ for free throws attempts (3.46 and 1.65 , respectively).

Table 2

Game efficiency variables achieved by female wheelchair basketball players from team ranking groups (A, B and C)

\begin{tabular}{|c|c|c|c|c|c|c|c|c|}
\hline \multirow{2}{*}{$\begin{array}{c}\text { Game } \\
\text { efficiency } \\
\text { variables }\end{array}$} & \multicolumn{2}{|c|}{$\mathrm{A}$} & \multicolumn{2}{|c|}{ B } & \multicolumn{2}{|c|}{$\mathrm{C}$} & \multicolumn{2}{|c|}{ TOTAL } \\
\hline & $M$ & $S D$ & $M$ & $S D$ & $M$ & $S D$ & $M$ & $S D$ \\
\hline TP & 10.77 & 7.36 & 8.87 & 6.16 & 6.41 & 4.74 & 9.33 & 6.72 \\
\hline $\mathrm{TSa}$ & 15.87 & 8.42 & 13.46 & 7.68 & 12.84 & 7.83 & 14.59 & 8.12 \\
\hline TS\% & 36.14 & 15.51 & 31.71 & 12.09 & 24.39 & 11.21 & 32.42 & 14.46 \\
\hline $\mathrm{BSa}$ & 6.39 & 3.74 & 4.97 & 3.42 & 4.65 & 2.74 & 5.65 & 3.51 \\
\hline $\mathrm{BS} \%$ & 43.96 & 22.10 & 37.32 & 16.68 & 30.88 & 17.57 & 39.39 & 20.37 \\
\hline $\mathrm{S} 2 \mathrm{a}$ & 11.20 & 5.84 & 10.05 & 5.15 & 10.11 & 6.19 & 10.67 & 5.70 \\
\hline $\mathrm{S} 2 \%$ & 37.51 & 17.40 & 29.08 & 16.84 & 24.81 & 11.76 & 32.58 & 16.87 \\
\hline $\mathrm{S} 3 \mathrm{a}$ & 0.53 & 1.18 & 1.77 & 4.92 & 0.50 & 1.07 & 0.83 & 2.66 \\
\hline $\mathrm{S} 3 \%$ & 9.40 & 21.29 & 7.11 & 11.98 & 2.39 & 5.29 & 7.07 & 16.69 \\
\hline FTa & 3.46 & 2.70 & 1.65 & 1.40 & 2.22 & 1.85 & 2.73 & 2.38 \\
\hline FT\% & 33.87 & 19.31 & 25.59 & 22.16 & 21.81 & 18.60 & 29.03 & 20.28 \\
\hline ST & 1.28 & 1.08 & 1.41 & 1.06 & 0.78 & 0.67 & 1.20 & 1.01 \\
\hline AS & 2.04 & 1.81 & 2.32 & 1.33 & 1.81 & 3.20 & 2.06 & 2.08 \\
\hline BL & 0.15 & 0.27 & 0.04 & 0.10 & 0.07 & 0.14 & 0.10 & 0.22 \\
\hline $\mathrm{OR}$ & 1.92 & 1.81 & 1.84 & 1.06 & 1.55 & 0.94 & 1.82 & 1.48 \\
\hline $\mathrm{DR}$ & 5.63 & 3.88 & 4.22 & 3.33 & 4.48 & 3.64 & 5.02 & 3.70 \\
\hline TO & 2.02 & 1.21 & 2.75 & 1.23 & 2.98 & 1.36 & 2.41 & 1.30 \\
\hline FA & 0.24 & 0.32 & 0.29 & 0.53 & 0.29 & 0.49 & 0.26 & 0.41 \\
\hline $\mathrm{DO}$ & 0.25 & 0.43 & 0.13 & 0.22 & 0.10 & 0.17 & 0.18 & 0.34 \\
\hline $\mathrm{CBGS}_{\text {modified }}$ & 37.93 & 34.77 & 21.35 & 25.92 & 8.91 & 22.00 & 27.34 & 32.23 \\
\hline
\end{tabular}


Note:

$A$ - teams from $1^{\text {st }}$ to $4^{\text {th }}$ place, $B$ - teams from $5^{\text {th }}$ and $6^{\text {th }}$ place, $C$ - teams from $7^{\text {th }}$ and $8^{\text {th }}$ place; TP - total points scored, TSa - total shots attempt, TS\% - , BSa - shots from 3 second area attempt, BS\% - , S2a - two-point shots attempt, S2\% - , S3a - three-point shots attempt, S3\% - , FTa - free throws attempts, FT\% - , ST - steals, AS - assists, BL - blocked shots, OR - offensive rebounds, DR defensive rebounds, TO - turnovers, FA - points scored after fast break, DO - points scored directly after offensive rebounds by rebounder, CBGS $S_{\text {modified }}$ - modification of Comprehensive Basketball Grading System.

Significant relationships between individual athletes' $\mathrm{CBGS}_{\text {modified }}$ score and 15 of the 19 measured variables were found, see table 3 .

In particular strong relationships were observed for $\mathrm{CBGS}_{\text {modified }}$ and defensive rebounds, total points scored, efficiency of total shoots, shots attempts from three seconds area, free throw shots attempts, twopoint shots attempts and efficiency of twopoints shots (from $r=.78$ to $r=.61$, respectively).

\section{Table 3}

Spearman rank order correlation coefficients between game efficiency variables and $\mathrm{CBGS}_{\text {modified }}$ values.

\begin{tabular}{|l|l|}
\hline \multicolumn{1}{|c|}{$\begin{array}{c}\text { Game } \\
\text { efficiency variables }\end{array}$} & CBGS modified values \\
\hline TP & $.72 *$ \\
\hline TSa & $.59 *$ \\
\hline TS\% & $.70^{*}$ \\
\hline BSa & $.69 *$ \\
\hline BS\% & $.59 *$ \\
\hline S2a & $.61 *$ \\
\hline S2\% & $.61 *$ \\
\hline S3a & .18 \\
\hline S3\% & .17 \\
\hline FTa & $.63 *$ \\
\hline FT\% & $.57 *$ \\
\hline ST & $.54 *$ \\
\hline AS & $.56 *$ \\
\hline BL & $.47 *$ \\
\hline OR & $.56 *$ \\
\hline DR & $.76^{*}$ \\
\hline TO & .15 \\
\hline FA & .15 \\
\hline DO & .49 \\
\hline
\end{tabular}

Note: TP - total points scored, TSa - total shots attempt, TS\% - , BSa - shots from 3 second area attempt, BS\% - , S2a-two-point shots attempt, $S 2 \%$ - , S3a - three-point shots attempt, S3\% - , FTa - free throws attempts, FT\% - , ST - steals, AS - assists, BL - blocked shots, OR - offensive rebounds, DR defensive rebounds, TO - turnovers, FA points scored after fast break, DO - points scored directly after offensive rebounds by rebounder, CBGS $S_{\text {modified }}$ - modification of Comprehensive Basketball Grading System; * $p<.05$.

One-way analysis of variance (ANOVA) indicated significant differences among the eight classes $(1-4.5)$ in total points scored, $F(7,64)=11.82, p=.000$; total shots attempt, $F(7,64)=11.67, p=.000$; shots from 3 second area attempt, $F(7,64)=9.40, p=$ .000 ; two-point shots attempt, $F(7,64)=$ $10.45, p=.000$; free throws attempts, $F(7,64)$ $=5.44, p=.000$; free throw percentage, $F(7$, $64)=3.23, p=.006$; steals, $F(7,64)=4.39, p$ $=.000$; assists, $F(7,64)=2.44, p=.028$; offensive rebounds, $F(7,64)=4.05, p=.001$; defensive rebounds, $F(7,64)=15.33, p=$ .000 ; turnovers, $F(7,64)=4.62, p=.000$; and $\mathrm{CBGS}_{\text {modified, }} F(7,64)=9.95, p=.000$. 


\section{Tabel 4}

Results of game efficiency variables achieved by wheelchair basketball players by functional level (classification)

\begin{tabular}{|c|c|c|c|c|c|c|c|c|c|c|c|c|c|c|c|c|}
\hline & \multicolumn{2}{|c|}{$\overline{0}$} & \multicolumn{2}{|c|}{1.5} & \multicolumn{2}{|c|}{2.0} & \multicolumn{2}{|c|}{2.5} & \multicolumn{2}{|c|}{3.0} & \multicolumn{2}{|c|}{3.5} & \multicolumn{2}{|c|}{4.0} & \multicolumn{2}{|c|}{4.5} \\
\hline & $\bar{M}$ & $S D$ & $\bar{M}$ & $S D$ & $M$ & $S D$ & $M$ & $S D$ & $M$ & $S D$ & $M$ & $S D$ & $M$ & $S D$ & $M$ & $S D$ \\
\hline $\mathrm{P}$ & 2.07 & 2.21 & 4.96 & 3.66 & 6.05 & 3.25 & 8.75 & 5.43 & 8.48 & 4.55 & 14.41 & 7.66 & 14.08 & 5.91 & 16.08 & 4.00 \\
\hline a & 5.01 & 2.63 & 9.28 & 4.25 & 11.51 & 7.89 & 14.81 & 6.34 & 14.97 & 5.92 & 20.36 & 8.19 & 19.59 & 3.86 & 22.47 & 6.88 \\
\hline $5^{\circ}$ & 3.39 & 22.57 & 29.21 & 14.35 & 28.59 & 7.10 & 30.81 & 9.23 & 27.61 & 6.91 & 38.35 & 8.35 & 40.29 & 15.64 & 40.06 & 7.90 \\
\hline$a$ & 1.78 & 1.09 & 3.41 & 1.81 & 4.78 & 1.93 & 5.57 & 2.07 & 5.50 & 3.55 & 9.36 & 3.81 & 8.19 & 3.24 & 7.93 & 3.03 \\
\hline & 31.84 & 32.84 & 34.09 & 21.94 & 34.12 & 10.47 & 33.03 & 17.75 & 39.83 & $\overline{9.25}$ & 44.80 & 7.69 & 51.10 & 15.46 & 46.73 & 6.65 \\
\hline & 3.76 & 2.33 & 7.09 & 2.83 & 8.22 & 4.35 & 11.24 & 3.92 & 12.08 & 56 & 14.34 & 4.9 & 15.32 & 3.2 & 14.63 & 5.0 \\
\hline$\%$ & 23.38 & 26.99 & 26.09 & 19.03 & 30.40 & 6.25 & 31.91 & 10.64 & 34.57 & 5.68 & 40.96 & 6.92 & 40.40 & 16.59 & 37.00 & 13.52 \\
\hline 3 & 0.00 & 0. & 0.26 & 0.63 & 0.84 & 1.55 & 0.50 & 1.0 & 0.70 & 1.21 & $\overline{0.33}$ & 0.55 & 0.77 & $\overline{1.2}$ & 2.80 & $\overline{5.9}$ \\
\hline $3 \%$ & 0.00 & 0.0 & 2.02 & 06 & 23.75 & 43.39 & 4.3 & 9. & 4.17 & 8.33 & 20.83 & 25.00 & 2.16 & 4.5 & 11.77 & 15.3 \\
\hline$=$ & 0.83 & 1. & 1.93 & 1.59 & 1.63 & 0.61 & 2.84 & 2.5 & 1.23 & 0.75 & 4.84 & 2.76 & 3.52 & 2.04 & 4.71 & 2.4 \\
\hline$\%$ & 3.33 & 18.92 & 33.67 & 17.11 & 25.36 & 16.08 & 27.89 & 22.00 & 8.00 & 10.95 & 30.83 & 16.88 & 38.17 & 17.86 & 41.07 & 18.8 \\
\hline & 0.23 & $\overline{0.4}$ & 0.79 & 0.74 & 0.82 & 0.71 & 1.16 & $\overline{0 .}$ & 1.77 & 1.03 & 1.74 & 1.00 & 1.70 & 0.98 & $1.7 \mathrm{~S}$ & $\overline{1.1}$ \\
\hline AS & 0.80 & 1.0 & 1.39 & 77 & 1.47 & 1.98 & 2.22 & 1.9 & 0.99 & 0.59 & 2.65 & 0.9 & 3.14 & 3.8 & 3.30 & 1.78 \\
\hline $3 \mathrm{~T}$ & 0.02 & 0.06 & 0.03 & 0.10 & 0.00 & 0.00 & 0.02 & 0.06 & 0.00 & 0.00 & 0.19 & 0.26 & 0.17 & 0.21 & 0.33 & 0.3 \\
\hline$\overline{\mathrm{OR}}$ & 0.62 & 0.66 & 1.12 & 0.58 & 1.40 & 0.63 & 1.60 & $\overline{0.99}$ & 1.91 & 1.11 & 2.95 & 1.75 & 2.49 & 1.04 & 2.81 & $\overline{2.2 !}$ \\
\hline$\overline{\mathrm{DR}}$ & 1.44 & 1.27 & 3.04 & 1.59 & 3.05 & 1.22 & 3.73 & 2.34 & 3.26 & 1.45 & 7.16 & 4.41 & 9.55 & 2.63 & 8.29 & $\overline{2.9}$ \\
\hline TO & 0.97 & 0.82 & 2.30 & 1.49 & 2.45 & 0.89 & 2.50 & 1.05 & 2.92 & 1.38 & 2.33 & 0.57 & 3.53 & 1.02 & 2.72 & 1.28 \\
\hline $\mathrm{FA}$ & 0.09 & 0.16 & 0.11 & 0.25 & 0.30 & 0.26 & 0.41 & 0.69 & 0.70 & 0.71 & 0.47 & 0.27 & 0.19 & 0.23 & 0.17 & 0.21 \\
\hline$\overline{\mathrm{DO}}$ & 0.00 & 0.00 & 0.00 & 0.00 & 0.04 & 0.09 & 0.15 & 0.32 & 0.23 & 0.41 & 0.41 & 0.46 & 0.38 & 0.54 & 0.31 & 0.30 \\
\hline $\mathrm{CBGS}$ & 3.10 & 13.24 & 8.70 & 9.47 & 7.35 & 12.54 & 17.82 & 26.29 & 8.16 & 16.79 & 55.38 & 38.59 & 54.61 & 32.47 & 57.00 & 24.6 \\
\hline
\end{tabular}

Note:

TP - total points scored, TSa - total shots attempt, TS\% - , BSa - shots from 3 second area attempt, BS\% - , S2a - two-point shots attempt, S2\% -, S3a - three-point shots attempt, S3\% - , FTa - free throws attempts, FT\% - , ST - steals, AS - assists, BL - blocked shots, OR - offensive rebounds, DR - defensive rebounds, TO - turnovers, FA - points scored after fast break, DO - points scored directly after offensive rebounds by rebounder, CBGS modified $_{-}$modification of Comprehensive Basketball Grading System.

\section{Table 5}

Differences between players functional levels (classification) in game efficiency variables (the post hock Tamhane's analysis)

\begin{tabular}{|c|c|c|c|c|c|c|c|c|c|c|c|c|c|c|c|c|c|c|c|c|c|c|c|c|c|c|c|c|}
\hline & \begin{tabular}{|l|}
1.0 \\
vs. \\
1.5 \\
\end{tabular} & \begin{tabular}{|l}
1.0 \\
vs. \\
2.0 \\
\end{tabular} & \begin{tabular}{|l}
1.0 \\
$v s$. \\
2.5 \\
\end{tabular} & \begin{tabular}{|l}
1.0 \\
$v s$. \\
3.0 \\
\end{tabular} & \begin{tabular}{|l|l|}
1.0 \\
vs. \\
3.5 \\
\end{tabular} & \begin{tabular}{|l}
1.0 \\
vs. \\
4.0 \\
\end{tabular} & \begin{tabular}{|l|}
1.0 \\
vs. \\
4.5 \\
\end{tabular} & \begin{tabular}{|l}
1.5 \\
vs. \\
2.0 \\
\end{tabular} & \begin{tabular}{|l}
1.5 \\
vs. \\
2.5 \\
\end{tabular} & $\begin{array}{l}1.5 \\
v s . \\
3.0 \\
\end{array}$ & \begin{tabular}{|l|}
1.5 \\
vs. \\
3.5 \\
\end{tabular} & $\begin{array}{l}1.5 \\
\text { vs. } \\
4.0 \\
\end{array}$ & \begin{tabular}{|l|}
1.5 \\
$v s$. \\
4.5 \\
\end{tabular} & \begin{tabular}{|l|}
2.0 \\
vs. \\
2.5 \\
\end{tabular} & \begin{tabular}{|l|}
2.0 \\
vs. \\
3.0 \\
\end{tabular} & \begin{tabular}{|l|}
2.0 \\
vs. \\
3.5 \\
\end{tabular} & \begin{tabular}{|l|}
2.0 \\
vs. \\
4.0 \\
\end{tabular} & \begin{tabular}{|l|}
2.0 \\
vs. \\
4.5 \\
\end{tabular} & \begin{tabular}{|l|}
2.5 \\
vs. \\
3.0 \\
\end{tabular} & \begin{tabular}{|l|}
2.5 \\
vs. \\
3.5 \\
\end{tabular} & \begin{tabular}{|l|}
2.5 \\
vs. \\
4.0 \\
\end{tabular} & \begin{tabular}{|l}
2.5 \\
vs. \\
4.5 \\
\end{tabular} & \begin{tabular}{|l|}
3.0 \\
vs. \\
3.5 \\
\end{tabular} & \begin{tabular}{|l|}
3.0 \\
vs. \\
4.0 \\
\end{tabular} & \begin{tabular}{|l|}
3.0 \\
vs. \\
4.5 \\
\end{tabular} & \begin{tabular}{|l|}
3.5 \\
vs. \\
4.0 \\
\end{tabular} & \begin{tabular}{l|}
3.5 \\
vs. \\
4.5 \\
\end{tabular} & \begin{tabular}{|l|}
4.0 \\
$v s$. \\
4.5 \\
\end{tabular} \\
\hline$\overline{\mathrm{TP}}$ & & & & & & * & 8 & & & & & 8 & 8 & & & & & 8 & & & & * & & & , & & & \\
\hline TSa & & & * & & & * & $\%$ & & & & & 8 & $\%$ & & & & & & & & & & & & & & & \\
\hline TS $\%$ & & & & & & & & & & & & & & & & & & & & & & & & & & & & \\
\hline$\overline{\mathrm{BSa}}$ & & & $*$ & & & * & 8 & & & & & 3 & $\%$ & & & & & & & & & & & & & & & \\
\hline $\mathrm{BS} \%$ & & & & & & & & & & & & & & & & & & & & & & & & & & & & \\
\hline $\mathrm{S} 2 \mathrm{a}$ & & & $*$ & & & * & $\%$ & & & & & \% & 8 & & & & & & & & & & & & & & & \\
\hline $\mathrm{S} 2 \%$ & & & & & & & & & & & & & & & & & & & & & & & & & & & & \\
\hline$\overline{S 3 a}$ & & & & & & & & & & & & & & & & & & & & & & & & & & & & \\
\hline S3\% & & & & & & & & & & & & & & & & & & & & & & & & & & & & \\
\hline FTa & & & & & & & $*$ & & & & & & & & & & & 8 & & & & & & & $\%$ & & & \\
\hline FT $\%$ & & & & & & & & & & & & & & & & & & & & & & & & 8 & 8 & & & \\
\hline ST & & & & & & * & $\%$ & & & & & & & & & & & & & & & & & & & & & \\
\hline AS & & & & & & & * & & & & & & & & & & & & & & & & & & $\%$ & & & \\
\hline $\mathrm{BL}$ & & & & & & & 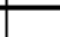 & & & & & & & & & & & & & & & & & & & & & \\
\hline$\overline{\mathrm{OR}}$ & & & & & & * & & & & & & & & & & & & & & & & & & & & & & \\
\hline$\overline{D R}$ & & & & & & * & $*$ & & & & & $\%$ & $\%$ & & & & $*$ & 8 & & & * & * & & \% & $\%$ & & & \\
\hline TO & & & * & & * & * & * & & & & & & & & & & & & & & & 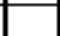 & & & & & & \\
\hline$\overline{\mathrm{FA}}$ & & & & & & & & & & & & & & & & & & & & & & & & & & & & \\
\hline$\overline{\mathrm{DO}}$ & & & & & & & & & & & & & & & & & & & & & & & & & & & & \\
\hline $\begin{array}{l}\text { CBGS } \\
\text { modifiod }\end{array}$ & & & & & & * & * & & & & & $*$ & $\%$ & & & & $*$ & 8 & & & & * & & & $\%$ & & & \\
\hline
\end{tabular}

Note:

TP - total points scored, TSa - total shots attempt, TS\% - , BSa - shots from 3 second area attempt, BS\% - , S2a - two-point shots attempt, S2\% -, S3a - three-point shots attempt, S3\% - , FTa - free throws attempts, FT\% - , ST - steals, AS - assists, BL - blocked shots, OR - offensive rebounds, DR - defensive rebounds, TO - turnovers, FA - points scored after fast break, DO - points scored directly after offensive rebounds by rebounder, CBGS modified - modification of Comprehensive Basketball Grading System; * $p<.05$. 


\section{DISCUSSION}

The results presented in his study showed that the game efficiency of elite female athletes depends on both the functional classification level and the teams ranking. Players from teams who ranked higher in Gold Cup 2006 showed higher levels of game efficiency. However, post-hoc analyzes did not reported significant relationships between all classes and team ranking groups.

There were many similarities in game efficiency between low-point players (functional classifications 1 to 3 ), and highpoint players (3.5 to 4.5 ).

Large inter-player variability for each variable and relatively small sample sizes may explain the lack of expected differences between both team ranking group and functional classification level. It is strongly recommended that for any future study an emphasis to recruit a larger sample size be made. However, we did use all of the eligible athletes attending these world championships. Vanlandewijck et al. (2003, 2004) had a similar sample size problem so they decided to considered "half-point" athletes to belong to the class below. However, functional levels $1.5,2.5$, and 3.5 , by IWBF regulations, are considered special cases. These "half-point" athletes present with functional capacities of each of the two neighbors' main classes (for example an athlete classed as 1.5 will have some functional characteristic of class 1 and 2) (Courbarioux, 1996). During Gold Cup 2006 the number of "half-point" athletes was large. For example the number of 2.5 athletes was larger than 2.0 or $3.0(11,5$, and 6 , respectively). Thus, it was decided in the present study to analyze our data using of all eight functional levels, even with the risks associated with decreasing the power of the statistical analysis. Our concern with arbitrarily combining classification levels for the sole purpose of improving power is two fold. First it does not respect the philosophy and intent of the IWBF functional classification system. Secondly, any differences found would not necessarily truly reflect reality. If class 2.5 was combined with class 2 and no differences were found between the restructured classes 2 and 3 , it could be solely the result of those "half-point" athletes skewing the class 2 data.

Analysis of top athletes in this study allows for a better understanding of the athletics potential of female wheelchair basketball athletes. Vanlandewijck et al. (2004) found similarities in team scores (shots per game, number of fouls, turnovers) between men's and women's wheelchair basketball. However, a major difference between shot efficiency of field goal shots and free throws were demonstrated between men and women (both group participated in Gold Cup 1998). Brasile (1993) also reported differences in shot efficiency between 1992 women's Paralympic team to the 1986 USA men's team (Gold Cup). The current study allowed for a comparison of the game efficiency of female wheelchair basketball athletes from the 1998 and 2006 World Championships. Free throw efficiency was similar in 1998 and 2006 (ranging across classes from 20 to $39 \%$ and from 25 to $41 \%$, respectively), except classes 1 and 3 in 2006 (13\% and $8 \%$, respectively). The number of free throw attempts per athlete in both tournaments was similar (from 1.15 to 4.80 in 1998, and from 0.83 to 4.84 in 2006). The 2006 games recorded better shooting efficiency from 3 second area (two-points inside) in comparison to 1998 (from $31.84 \%$ to $51.10 \%$ in 2006 , and from $9.92 \%$ to $31.89 \%$ in 1998). Given that there have not been any regulatory changes that would affect shooting, this higher shooting efficiency suggests an improvement in the level of sport skill proficiency in women's wheelchair basketball since the 1998 tournament. The similar number of shots from inside the key (from 1.13 to 9.07 in 1998, and from 1.78 to 9.36 in 2006) indicates that the decrease the shot clock time from $30 \mathrm{~s}$ to $24 \mathrm{~s}$ generally did not influence game performance or shooting efficiency. However, the number of shots taken demonstrated a new role for class 2 and 2.5 players. This higher number of shots in 2006 (4.78 - 5.57 versus 2.22) in comparison to Gold Cup 1998 provides some evidence that athletes with a classification of 
2 and 2.5 are spending more time in the game with the ball. Besides improved fitness and skills, it is possible that the liberalization of classification system which allows more strapping without changing ones classification level has contributed to altered role of classes 2 and 2.5 on the court.

Women's wheelchair basketball tends to be a low scoring game. The general limited functional capacity of women class 1.0 athletes tends to restrict their ability to shoot and be an offensive threat during the game. Across the entire pool of athlete's evaluated, the average of shots per game was 1 in both the 1998 and 2006 tournaments, except those in class 4.5. These higher functioning athletes' average just under 3 shots per game. However, still shooting attempts and efficiency is very low. The similarities in three-point shots between both tournaments reflect that these shots are an exception in female basketball as compared to the men. It seems that these shooting variables should be important in women's wheelchair basketball.

Comparison of offensive and defensive rebounds, blocked shots showed similarities between game efficiency in 1998 and 2006 among all classes. Smaller number of turnovers in 2006 indicated some improvements ball handling skill (from 2.17 to 4.58 in 1998, and $0.97-3.53$ in 2006). All of these variables generally confirm higher level of skill performance or athleticism (i.e. better technique abilities, experience, coaching system) in female wheelchair basketball athletes.

In both articles by Vanlandewijck et al. (2003, 2004), the two-way analysis of variance used modified functional classification categories and player position as main factors. In the present study objective description of players' position was not possible. Misunderstandings in clear definitions of a players' position were found between our basketball experts. Some coaches used different positioning system of players. Moreover, athletes are able to change their positions throughout the game.

Interpretation of fouls is still too difficult to objectively evaluate. Although some fouls seems to be a result of significant differences in players' performance, skills and experience, in many cases players make 'intentional' fouls, thus making the analysis very difficult.. Fouling during that game is now part of the strategy and philosophy of many coaches. These types of fouls are used to stop an attack or for intimidation and do not reflect an inadequate or lower skill of the athlete being observed. Given that the recognition of these different types of fouls is not practical in the context of individual athlete evaluation, these authors elected not to include fouls as part of the analysis.

Further development of the wheelchair basketball observational game efficiency scoring form is still needed. In the present study many game efficiency variables were analyzed but only three were related to defensive actions - steals, blocked shots, and defensive rebounds. We recommend the continuation of the development of these observational forms based also on games variables without the ball. Although an important part of the game, there is too much potential misunderstanding in interpretation game actions such as: affective screens, wheelchair positioning, picking forecourt and backcourt, blocking out during rebounding, assisting or helping another partners in defense. This challenge in evaluating game efficiency is typical for other Olympic and Paralympic team games i.e. standing basketball or wheelchair rugby (Huciński \& Tymański, 2008; Molik et al., 2008). In the present study new variables of game efficiency were included - points scored after fast break, and points scored directly after offensive rebounds by rebounder. Impressively the inter-observer reliability for these variables was high $(r>.85)$.

The greatest number of differences found between functional classes was for rebounding. Rebounding is an action where trunk stability plays an critical role. Better trunk stabilization increases volume of action and therefore influences number of effective rebounds. It seems that trunk stabilization could be a main factor that differentiates functional levels in wheelchair basketball. 
Molik and Kosmol (2001) based on an analysis of Polish male wheelchair basketball players also reported large differences between classes in numbers of rebounds.

The aim of this study was not the evaluation of the IWBF functional classification system. However, similarities and differences between functional classes in game efficiency could provide valuable evidence to help reevaluate the current system or establish a new classification system.

Unfortunately, our results were unable to identify any significant differences between neighboring (e.g., classes 2 and 3) functional levels. Similarities between low-point players from 1 to 3 demonstrate that this group of athletes performs at similar levels of skill. However a small sample size may have minimized many potentially import differentiating results. The identified differences between classes 1 and 2.5 suggests the unique nature of class 1.0 in terms of performance. It is clear to those involved with wheelchair basketball, scientists, coaches, and classifiers, that class 1 athletes have significantly lower functional capabilities as compared to athletes of higher classes. In women's wheelchair baskeball these players are often given the role of guard versus a center or forward, but without the ball handling requirements. In our opinion this positioning is not effective for the player or the team. Even if the player is tall they should use a low riding wheelchair (significant seat dump, smaller wheels, and/or larger camber) and a high backrest (Yilla, Bar, \& Dangelmaier, 1998).

Interesting there were also similarities between classes 3.5, 4, and 4.5. Why 3.5 achieved similar game efficiency level in comparison to classes $4-4.5$ as compared to other authors who would have collapsed classification levels and placed these athletes in class 3? In our opinion trunk stability in frontal plane is the main factor. Class 3.5 players in comparison to other lower classes are able to make any movement in frontal plane, though still limited. This movement significantly increases the volume of action particularly during rebounding. This data shows that along with those athletes in class 4 and 4.5 , athletes in class 3.5 can be effective in the center position.

Differences between players based on team ranking were identified in shooting efficiency in total shots, two-point shots, and in CBGS $_{\text {modified }}$ values. These differences were reported to be significant between the first four, and last two teams of the 2006 tournament, groups A and C. Our results showed that shooting efficiency is one of the main factors that would help raise the level of game efficiency in women's wheelchair basketball. Differences in free throws variables while intuitively important between teams from $1^{\text {st }}$ to $4^{\text {th }}$ places, and $5^{\text {th }}$ and $6^{\text {th }}$ places in ranking were too variable in this study for an objective interpretation.

Spearman rank order correlation coefficients identified significant relationships between classification levels and all variables, except points scored during a fast break. It seems that this particular variable is not common as in women' wheelchair basketball as one would expect.

The present analyzes did not show significant relationships between three-point shots, turnovers, and points scored during a fast break, and $\mathrm{CBGS}_{\text {modified }}$ values. Points scored during a fast break and three-point shot attempts are still not typical in women' wheelchair basketball. Though unlikely, lack of significant differences could also be a result of improper calculation or misinterpretation these elements in game efficiency. It is recommended that the CBGS $_{\text {modified }}$ system be reexamine for any future study. A reevaluation of the relative weighting of variables, the inclusion of "older" variables and the consideration of the inclusion of new more objective wheelchair basketball specific assessment variables. This new system could better evaluate quality of game of low-point players who prefer or are coached in a system where they often play without the ball. This same concern of adequately assessing the contribution of the lower-point players has also come up in wheelchair rugby (Molik et al., 2008). 


\section{SUMMARY}

Both research hypotheses were confirmed in present study. Analyses of the results supports that game efficiency in women's wheelchair basketball is dependent on the athlete's functional level and overall team ranking. However, there are many similarities in game efficiency between those athletes in adjacent classifications levels.

The results of this study supports the notion that athletes from "best" teams demonstrate higher shooting efficiency and significantly better $\mathrm{CBGS}_{\text {modified }}$ in comparison to players from the "weakest" teams. Of the variables observed there are a number that were observed on a limited bases such as: three-point shots, points scored during a fast break, points scored directly after offensive rebounds by rebounder and blocked shots. Development these important skills would have a significant impact on the improvement of female athletes' level of wheelchair basketball.

\section{REFERENCES}

Brasile, F. M. (1990). Performance Evaluation of Wheelchair Athletes: More than a Disability Classification Level Issue. Adapted Physical Activity Quarterly, 7(4), 289-297.

Brasile, F. M. (1993). Evaluating the elite. Sport'n Spokes, 19(3), 52-55.

Brasile, F.M. \& Hedrick, B.N. (1996). The relationship of skills of elite wheelchair basketball competitors to the international functional classification system. Therapeutic Recreation Journal, 30(2), 114127.

Byrnes, D., \& Hedrick, B. (1994). Comprehensive basketball grading system. In B. Hedrick, D. Byrnes, \& L. Shaver, Wheelchair Basketball (p.79). Washington, DC: Paralyzed Veterans of America.

Courbariaux, B. (1996). The Classification system for wheelchair basketball players. International Wheelchair Basketball Federation. Published and distributed by the IWBF.
Doyle, T.L.A., Davis, R.W., Humphries, B., Dugan, E.L., Horn, B.G., Shim, J.K., \& Newton, R.U. (2004). Further Evidence to Change the Medical Classification System of The National Wheelchair Basketball Association. Adapted Physical Activity Quarterly, 21(1), 63-70.

Huciński, T. \& Tymański, R. (2008). The Observation Sheet Method in the Assessment Process. Research Yearbook, 14(2), 65-68.

Hutzler, Y., Ochana, S., Bolotin, R. \& Kalina, E. (1998). Aerobic and anaerobic arm cranking Power outputs of males with lower limb impairments: Relationship with sport participation intensity, age, impairment and functional classification. Spinal Cord, 36(3), 205-212.

IWBF (2004). A Guide to the Functional Classification of Wheelchair Basketball Players. Prepared by the International Wheelchair Basketball Federation Player Classification Commission.

IWBF (2008). Official Wheelchair Basketball Rules 2008. As approved by International Wheelchair Basketball Federation Executive Council.

Malone, L.A., Gervais, P.L. \& Steadward, R.D. (2002). Shooting mechanics related to player classification and free throw success in wheelchair basketball. Journal of Rehabilitation Research and Development, 39(6), 701-710.

Molik, B. \& Kosmol, A. (2001). In search of objective criteria in wheelchair basketball player classification. In G. Doll-Tepper, M. Kröner, \& W. Sonnenschein (Eds.), Vista'99 - New horizons in sport for athletes with a disability: Proceedings of the international Vista'99 conference (pp. 355-368) Köln, Germany: Meyer \& Meyer Sport.

Molik, B., Kosmol, A., \& Rutkowska, I. (2005) Anaerobic performance as a criterion in classifying wheelchair basketball players. Movement and Health $4^{\text {th }}$ International Conference. Proceedings (Abstract and full papers). Olomouc, November 23-25, 2005. CD-ROM. 
Molik, B., Lubelska, E., Kosmol, A., Bogdan, M., Yilla, A. B., \& Hyla, E. (2008). An Examination of the International Wheelchair Rugby Federation Classification System Utilizing Parameters of Offensive Game Efficiency. Adapted Physical Activity Quarterly, 25(4), 335-351.

Pszczolowski, T. (1978) Mała encyclopedia prakseoligii I teorii organizacji. Wrocław, Zakład Narodowy im. Ossolinskich.

Rotstein, A., Sagiv, M, Ben-Sira, D., Werber, G., Hutzler, J., \& Annenburg, H. (1994). Aerobic capacity and anaerobic threshold of wheelchair basketball players, Paraplegia, 32(3), 196-201.

Schmid, A., Huonker, M., Stober, P., Barturen, J. M., Schmidt-Trucksass, A., Durr, H., Volper, H. J., \& Keul, J. (1998). Physical performance and cardiovascular and metabolic adaptation of elite female wheelchair basketball players in wheelchair ergometry and in competition. American Journal of Physical Medicine \& Rehabilitation, 77(6), 527-533.

Vanlandewijck, Y. C., Spaepen, A. J., \& Lysens R. J. (1995). Relationship Between the Level of Physical Impairment and Sport Performance in Elite Wheelchair Basketball Athletes. Adapted Physical Activity Quarterly, 12(2), 139-150.

Vanlandewijck, Y.C., Evaggelinou, Ch., Daly, D.D., Van Houtte, S., Verellen, J., Aspeslagh, V., Hendrickx, R., Piessens, T., \& Zwakhoven, B. (2003). Proportionality in Wheelchair Basketball Classification. Adapted Physical Activity Quarterly, 20(4), 369-380.

Vanlandewijck, Y.C., Evaggelinou, Ch., Daly, D.D., Verellen, J., Van Houtte, S., Aspeslagh, V., Hendrickx, R., Piessens, T., \& Zwakhoven, B.(2004). The relationship between functional potential and field performance in elite female wheelchair basketball players. Journal of Sports Sciences, 22(7), 668-675.

Yilla, A.B., La Bar, R.H., \& Dangelmaier, B.S. (1998). Setting Up Wheelchair for Basketball. Sport'n Spokes, 24(2), 63-65.

Corresponding author's e-mail address: b_molik@poczta.onet.pl

\section{SPIELEFFIZIENZ VON WEIBLICHEN ELITE-ROLLSTUHLBASKETBALL- SPIELERINNEN WÄHREND DER WELTMEISTERSCHAFT (GOLD CUP) 2006}

(Resümee)

Die Ziele der Studie waren (1) die Beschreibung der Spieleffizienz der Teams, abhängig von der Rangliste im Gold Cup 2006 und (2) die Bestätigung der Relation zwischen den Klassifikationslevels der weiblichen Elite-Rollstuhlbasketball-Spielerinnen und ihrer Basketball Leistung. Eine Gruppe von 72 Athletinnen aus acht Teams, die an der Weltmeisterschaft in Rollstuhlbasketball 2006 (3.-16. Juli in Amsterdam, Niederlande) teilgenommen hatten, wurde für diese Studie ausgewählt. Alle vierundzwanzig angesetzten Spiele wurden per Video aufgezeichnet. Die weiblichen Athletinnen wurden nach ihrer funktionellen Klassifizierung (1, 1.5, 2, 2.5, 3, 3.5, 4, und 4.5) und ihrem Ranglistenplatz im Gold Cup (Teams vom 1. bis 4. Platz, Teams vom 5. bis 6. Platz, Teams vom 7. bis 8. Platz) eingeteilt. Neunzehn Variable der Spieleffizienz wurden festgehalten. Zur Feststellung der Qualität des Spielerbeitrags für das Spiel wurde eine modifizierte Version des Comprehensive Basketball Grading System (CBGS) angewendet. Die Analysen der Resultate bestätigen, dass die Spieleffizienz im Frauen-Rollstuhlbasketball vom funktionalen Level und von der Team-Rangliste abhängig ist. Dennoch gibt es viele Ähnlichkeiten unter den Athletinnen mit benachbarten Klassifikationslevels. Die Ergebnisse unterstützen die Annahme, dass 
Athletinnen von den „besten“ Teams eine höhere Treffer-Effizienz zeigen und signifikant bessere CBSG-Werte haben als weibliche Spielerinnen von „schwächeren“ Teams.

SCHLÜSSELWÖRTER: Behindertensport, Spieleffizienz, Klassifikation, Fertigkeiten, Rangliste.

\section{L'EFFICACITE DE JEU DE JOUEUSES ELITES DE BASKETBALL EN FAUTEUIL ROULANT DURANT LES CHAMPIONNATS DU MONDE (GOLD CUP) 2006}

(Résumé)

L'objectif de cette étude était de (1) décrire l'efficacité de jeu de plusieurs équipes selon leur classement à la Gold Cup 2006, et (2) de confirmer la relation entre la classification de niveaux de joueuses élites de basketball en fauteuil roulant et leur performance. Un groupe de 72 athlètes représentant huit équipes participants aux Championnats du Monde pour le basketball en fauteuil roulant en 2006 (3-16 juillet, Amsterdam, Pays-Bas) ont été sélectionnées pour participer à cette étude. Les 24 matchs ont été enregistrés. Les athlètes féminines ont été réparties selon leur niveau de classification fonctionnelle $(1,1.5,2,2.5,3,3.5$, 4, et 4.5$)$ et le classement de leur équipe dans la Gold Cup (les équipes de la $1^{\text {ère }}$ à la $4^{\mathrm{e}}$ position, les équipes en $5^{\mathrm{e}}$ et $6^{\mathrm{e}}$ position et les équipes en $7^{\mathrm{e}}$ et $8^{\mathrm{e}}$ position). Dix-neuf variables d'efficacité de jeu ont été évaluées. Pour déterminer la qualité de la contribution d'une athlète au jeu, une modification du CBGS (Comprehensive Basketball Grading System) a été utilisé. Les analyses des résultats soutiennent que l'efficacité de jeu dans le basketball en fauteuil roulant féminin dépend du niveau de classification fonctionnelle et du classement de leur équipe. Toutefois, il existe de nombreuses similarités entre les athlètes ayant des niveaux de classifications équivalents. Les résultats soutiennent la notion que les athlètes venants des «meilleures » équipes démontrent une meilleure efficacité au tir et présentent des valeurs de CBGS significativement plus élevées comparées aux joueuses féminines des équipes les plus «faibles ».

MOTS CLEFS: handisport, efficacité de jeu, classification, habiletés, classement. 